\title{
ON THE SUM OF DIGITS OF NUMERATORS OF BERNOULLI NUMBERS
}

\author{
ATTILA BÉRCZES AND FLORIAN LUCA
}

\begin{abstract}
Let $b>1$ be an integer. We prove that for most $n$, the sum of the digits in base $b$ of the numerator of the Bernoulli number $B_{2 n}$ exceeds $c \log n$, where $c:=c(b)>0$ is some constant depending on $b$.
\end{abstract}

2000 Mathematics Subject Classification: Primary 11B68

\section{IntroduCtion}

Let $\left\{B_{n}\right\}_{n \geq 0}$ be the sequence of Bernoulli numbers given by $B_{0}=1$ and

$$
B_{n}=1-\sum_{k=0}^{n-1}\left(\begin{array}{l}
n \\
k
\end{array}\right) \frac{B_{k}}{n-k+1} \quad \text { for all } n \geq 1 .
$$

Then $B_{1}=-1 / 2$ and $B_{2 n+1}=0$ for all $n \geq 0$. Furthermore, we have $(-1)^{n+1} B_{2 n}>0$. Write $B_{2 n}=:(-1)^{n+1} C_{n} / D_{n}$ with coprime positive integers $C_{n}$ and $D_{n}$. The denominator $D_{n}$ is well-understood by the von Staudt-Clausen theorem which asserts that

$$
D_{n}=\prod_{p-1 \mid 2 n} p
$$

Let $b>1$ be an integer. For a positive integer $m$, put $s_{b}(m)$ for the sum of digits of $m$ in base $b$. In [3], it was shown that there exists a positive constant $c_{0}$ depending on $b$ such that the inequality $s_{b}(n !)>c_{0} \log n$ holds for all positive integers $n$. Here, we prove that a similar inequality holds with $n$ ! replaced by $C_{n}$ on a set of $n$ of asymptotic density 1 .

The research was supported in part by project SEP-CONACyT 79685, by grants T67580 and T75566 of the Hungarian National Foundation for Scientific Research. The work is supported by the TÁMOP 4.2.1./B-09/1/KONV-2010-0007 project. The project is implemented through the New Hungary Development Plan, cofinanced by the European Social Fund and the European Regional Development Fund. F. L. worked on this project while he visited the Institute of Mathematics of the University of Debrecen, Hungary in August 2011. He thanks the members of that department for their hospitality. 
Theorem 1. The inequality

$$
s_{b}\left(C_{n}\right)>\frac{\log n}{6 \log b}
$$

holds on a set of positive integers $n$ of asymptotic density 1 .

In what follows, we use the Landau symbols $O$ and $o$ and the Vinogradov symbols $\gg$ and $\ll$ with their usual meaning. We also use $c_{0}, c_{1}, \ldots$ for computable positive constants and $x_{0}$ for a large real number, not necessarily the same from one occurrence to the next.

Proof. Consider the following set of positive integers:

$$
\mathcal{M}_{b}(x):=\left\{n \in[x / 2, x): s_{b}\left(C_{n}\right)<\frac{\log x}{6 \log b}\right\} .
$$

We need to show that $\# \mathcal{M}_{b}(x)=o(x)$ as $x \rightarrow \infty$, for after this the conclusion of Theorem 1 will follow by replacing $x$ by $x / 2$, then by $x / 4$, and so on, and summing up the resulting estimates.

Put $y:=\log x$ and consider the set

$$
\mathcal{L}(x):=\{n \in[x / 2, x): p-1 \mid 2 n \text { for some prime } p \geq y\} .
$$

It follows from Theorem 2 in [2] that

$$
\# \mathcal{L}(x)=o(x) \quad \text { as } \quad x \rightarrow \infty .
$$

We now put

$$
\mathcal{N}_{b}(x):=\mathcal{M}_{b}(x) \backslash \mathcal{L}(x) .
$$

In light of (4), it suffices to show that $\# \mathcal{N}_{b}(x)=o(x)$ as $x \rightarrow \infty$.

Let

$$
\mathcal{D}(x):=\left\{D_{n}: n \in \mathcal{N}_{b}(x)\right\} .
$$

Since $n \notin \mathcal{L}(x)$, it follows that if $p \mid C_{n}$, then $p<y$. Thus,

$$
\# \mathcal{D}(x) \leq 2^{\pi(y)}=x^{o(1)} \quad \text { as } \quad x \rightarrow \infty .
$$

For $n \in \mathcal{N}_{b}(x)$, we write

$$
C_{n}=d_{1} b^{n_{1}}+d_{2} b^{n_{2}}+\cdots+d_{s} b^{n_{s}},
$$

where $d_{1}, \ldots, d_{s} \in\{1, \ldots, b-1\}$ and $n_{1}>n_{2}>\cdots>n_{s}$. We put $t:=$ $t(n)$ for the smallest index $i \in\{1,2, \ldots, s-1\}$ such that $b^{n_{1}-n_{i+1}}>n^{2}$ if it exists and set $t:=s$ otherwise. From the definition of $t(n)$, we see immediately that

$$
\begin{aligned}
C_{n} & =\left(d_{1} b^{n_{1}}+\cdots+d_{t} b^{n_{t}}\right)\left(1+O\left(n^{-2}\right)\right) \\
& :=b^{m_{n}} E_{n}\left(1+O\left(x^{-2}\right)\right),
\end{aligned}
$$

where $m=m_{n}:=n_{t}$ and $E_{n}:=d_{1} b^{n_{1}-n_{t}}+d_{2} b^{n_{2}-n_{t}}+\cdots+d_{t}$. 
Let $\mathcal{E}_{b}(x)$ be the subset of all possible values for $E_{n}$ for $n \in \mathcal{N}_{b}(x)$. Let us find an upper bound for the cardinality of this set. Observe first that

$$
E_{n}<b^{n_{1}-n_{t}+1} \leq b^{2(\log x) /(\log b)+1} .
$$

The positive integers $E:=E_{n}$ bounded by the quantity appearing on the right-hand side of inequality $(10)$ have at most $K:=\lfloor 2(\log x) /(\log b)+$ $2\rfloor$ digits in base $b$. As $n \in \mathcal{N}_{b}(x)$, the number of nonzero digits of $E_{n}$ is bounded by $S:=\lfloor(\log x) /(6 \log b)\rfloor$, and the number of possible values for $E$ is at most

$$
\begin{aligned}
\sum_{i=0}^{S}\left(\begin{array}{c}
K \\
i
\end{array}\right)(b-1)^{i} & \leq(S+1)\left(\begin{array}{c}
K \\
S
\end{array}\right)(b-1)^{S} \leq(S+1)\left(\frac{(b-1) e K}{S}\right)^{S} \\
& \leq\left(\frac{\log x}{6 \log b}+1\right)\left(\frac{(b-1) e}{3}+o(1)\right)^{(\log x) /(6 \log b)}=x^{\delta+o(1)}
\end{aligned}
$$

as $x \rightarrow \infty$, where

$$
\delta:=\frac{\log ((b-1) e / 3)}{6 \log b}<\frac{1}{6}
$$

Thus, we get that

$$
\# \mathcal{E}_{b}(x)=x^{\delta+o(1)} \quad \text { as } \quad x \rightarrow \infty .
$$

We next use the formula

$$
\zeta(2 n)=(-1)^{n+1} B_{2 n} \frac{(2 \pi)^{2 n}}{2(2 n) !}=\frac{C_{n}(2 \pi)^{2 n}}{D_{n} 2(2 n) !},
$$

as well as the aproximation

$$
\zeta(2 n)=1+\frac{1}{2^{2 n}}+\frac{1}{3^{2 n}}+\cdots=1+O\left(\frac{1}{2^{2 n}}\right)
$$

to get that

$$
C_{n}=D_{n} \frac{2(2 n) !}{(2 \pi)^{2 n}} \zeta(2 n)=D_{n} \frac{2(2 n) !}{(2 \pi)^{2 n}}\left(1+O\left(\frac{1}{2^{2 n}}\right)\right) .
$$

We take logarithms in (12) above to arrive at

$\log C_{n}-\log D_{n}-\log (2(2 n) !)+2 n \log (2 \pi)=\log \left(1+O\left(\frac{1}{2^{2 n}}\right)\right)=O\left(\frac{1}{2^{x}}\right)$.

Taking logarithms in (9) and comparing the result with (13), we get

$$
\log (2(2 n) !)-2 n \log (2 \pi)=\log \left(E_{n} / D_{n}\right)+m_{n} \log b+O\left(\frac{1}{x^{2}}\right)
$$


Now fix a pair of numbers $(D, E) \in \mathcal{D}_{b}(x) \times \mathcal{E}_{b}(x)$ and look at the set

$$
\mathcal{N}_{b, D, E}(x):=\left\{n \in \mathcal{N}_{b}(x):\left(D_{n}, E_{n}\right)=(D, E)\right\} .
$$

We let $z:=x^{1 / 5}$ and show that if $x>x_{0}$, then every subinterval $I$ of $[x / 2, x]$ of length $z$ contains at most two elements of $\mathcal{N}_{b, D, E}(x)$. Well, assume that this is not so and let $n_{0}<n_{1}<n_{2}$ be all three in $\mathcal{N}_{b, D, E}(x)$, where $n_{2}-n_{0} \leq z$. Write $n_{i}=: n_{0}+k_{i}$ for $i=0,1,2$, with $0=k_{0}<k_{1}<k_{2} \leq z$. We evaluate relation (14) in $n:=n_{i}:=n_{0}+k_{i}$ for $i=0,1,2$ getting

$\log \left(2\left(2 n_{i}\right) !\right)+2 n_{i} \log (2 \pi)=\log (E / D)+m_{n_{i}} \log b+O\left(\frac{1}{x^{2}}\right), \quad(i=0,1,2)$.

We let $\Lambda:=\left(\lambda_{0}, \lambda_{1}, \lambda_{2}\right)$ be a nonzero vector of integers such that

$$
\left\{\begin{array}{cccc}
\lambda_{0} & +\lambda_{1}+\lambda_{2}= & 0 \\
n_{0} \lambda_{0} & + & n_{1} \lambda_{1} & + \\
n_{2} \lambda_{2} & = & 0
\end{array}\right.
$$

For example, we can put $\Lambda:=\left(k_{1}+k_{2},-k_{2},-k_{1}\right)$. With this choice,

$$
\max \left\{\left|\lambda_{i}\right|: i=0,1,2\right\} \leq 2 z .
$$

Note also that system (17) is equivalent to

$$
\left\{\begin{aligned}
\lambda_{0}+\lambda_{1}+\lambda_{2} & =0 \\
k_{1} \lambda_{1}+k_{2} \lambda_{2} & =0
\end{aligned}\right.
$$

Taking the linear combination of the three relations (16) with the coefficients given by $\Lambda$, we get

$$
\sum_{i=0}^{2} \lambda_{i} \log \left(2\left(2 n_{i}\right) !\right)=\Gamma \log b+O\left(\frac{z}{x^{2}}\right)
$$

where $\Gamma:=\sum_{i=0}^{2} \lambda_{i} m_{n_{i}} \in \mathbb{Z}$. Write

$$
2\left(2 n_{i}\right) !=2\left(2 n_{0}\right) !\left(2 n_{0}+1\right) \cdots\left(2 n_{i}\right)=: 2\left(2 n_{0}\right) ! X_{i}, \quad(i=0,1,2) .
$$

Hence,

$$
\begin{aligned}
\sum_{i=0}^{2} \lambda_{i} \log \left(2\left(2 n_{i}\right) !\right. & =\sum_{i=0}^{2} \lambda_{i}\left(\log \left(2\left(2 n_{0}\right) !\right)+\log X_{i}\right) \\
& =\sum_{i=0}^{2} \lambda_{i} \log \left(2\left(2 n_{1}\right) !\right)+\sum_{i=0}^{2} \lambda_{i} \log X_{i} \\
& =\sum_{i=0}^{2} \lambda_{i} \log X_{i}=\lambda_{1} \log X_{1}+\lambda_{2} \log X_{2}
\end{aligned}
$$


where in the above equalities we used the first equation of (17) as well as the fact that $X_{0}=1$. Writing

$$
\begin{aligned}
\log X_{i} & =\sum_{j=1}^{2 k_{i}} \log \left(2 n_{0}+j\right)=2 k_{i} \log \left(2 n_{0}\right)+\sum_{j=1}^{2 k_{i}} \log \left(1+\frac{j}{n_{0}}\right) \\
& =2 k_{i} \log \left(2 n_{0}\right)+\sum_{j=1}^{2 k_{i}}\left(\frac{j}{n_{0}}+O\left(\frac{j^{2}}{n_{0}^{2}}\right)\right) \\
& =2 k_{i} \log \left(2 n_{0}\right)+\sum_{j=1}^{2 k_{i}} \frac{j}{n_{0}}+O\left(\sum_{j=0}^{2 k_{i}} \frac{j^{2}}{n_{0}^{2}}\right) \\
& =2 k_{i} \log \left(2 n_{0}\right)+\frac{2 k_{i}\left(2 k_{i}+1\right)}{n_{0}}+O\left(\frac{k_{i}^{3}}{x^{2}}\right)
\end{aligned}
$$

for $i=1,2$, we get that

$$
\begin{aligned}
\lambda_{1} \log X_{1}+\lambda_{2} \log X_{2} & =2\left(\lambda_{1} k_{1}+\lambda_{2} k_{2}\right) \log \left(2 n_{0}\right)+\frac{4 \lambda_{1} k_{1}^{2}+4 \lambda_{2} k_{2}^{2}}{n_{0}} \\
& +\frac{2 \lambda_{1} k_{1}+2 \lambda_{2} k_{2}}{n_{0}}+O\left(\frac{k_{2}^{4}}{n_{0}^{2}}\right) \\
& =4\left(\frac{\lambda_{1} k_{1}^{2}+\lambda_{2} k_{2}^{2}}{n_{0}}\right)+O\left(\frac{z^{4}}{x^{2}}\right) .
\end{aligned}
$$

Inserting the above estimate into the left-hand side of (20), we get

$$
4\left(\frac{\lambda_{1} k_{1}^{2}+\lambda_{2} k_{2}^{2}}{n_{0}}\right)=\Gamma \log b+O\left(\frac{z}{x^{2}}+\frac{z^{4}}{x^{2}}\right)=\Gamma \log b+O\left(\frac{1}{x^{6 / 5}}\right) .
$$

If $\Gamma=0$, then the above estimate $(21)$ implies that

$$
\left|\lambda_{1} k_{1}^{2}+\lambda_{2} k_{2}^{2}\right|=O\left(\frac{n_{0}}{x^{6 / 5}}\right)=O\left(\frac{1}{x^{1 / 5}}\right)=o(1)
$$

as $x \rightarrow \infty$, showing that $\lambda_{1} k_{1}^{2}+\lambda_{2} k_{2}^{2}=0$ for $x>x_{0}$. This equation together with equations (19) shows that $\lambda_{1}=\lambda_{2}=\lambda_{3}=0$, which is false. Thus, $\Gamma \neq 0$, showing the right-hand side in estimate (21) is $\gg 1$, and since the left-hand side of estimate $(21)$ is $O\left(k_{2}^{3} / x\right)$, we get that $k_{2} \gg x^{1 / 3}$. This is not possible for large $x$ because $k_{2} \leq z$. We conclude that indeed for large $x, I$ cannot contain three numbers from $\mathcal{N}_{b, D, E}(x)$. This shows that

$$
\# \mathcal{N}_{b, D, E}(x) \leq\left[\frac{x-x / 2}{z}\right]+2 \ll x^{4 / 5} .
$$


Hence, by estimates (7) and (11), we get that

$$
\begin{aligned}
\mathcal{N}_{b}(x) & =\sum_{(D, E) \in \mathcal{D}_{b}(x) \times \mathcal{E}_{b}(x)} \# \mathcal{N}_{b, D, E} \ll x^{4 / 5} \times \# \mathcal{D}_{b}(x) \times \# \mathcal{E}_{b}(x) \\
& \leq x^{4 / 5+\delta+o(1)}<x^{29 / 30+o(1)}=o(x)
\end{aligned}
$$

as $x \rightarrow \infty$, which is what we wanted to prove.

\section{REFERENCES}

[1] A. Bérczes and F. Luca, "On the largest prime factor of numerators of Bernoulli numbers", Preprint, 2011.

[2] P. Erdős and S. S. Wagstaff Jr., "The fractional parts of the Bernoulli numbers", Illinois J. Math. 24 (1980), 104-112.

[3] F. Luca: "The number of non-zero digits of $n$ !", Canadian Math. Bull. 45 (2002), 115-118.
A. BÉRCZes
Institute of Mathematics, University of DeBrecen
AND University of DeBReCEN
H-4010 Debrecen, P.O. Box 12, Hungary
E-mail address: berczesa@math.klte.hu
F. LUCA
INSTITUTO DE MATEMÁticAS
Universidad Nacional Autonoma de México
C.P. 58089, Morelia, Michoacán, México
E-mail address: fluca@matmor.unam.mx

Number Theory Research Group, Hungarian Academy of Sciences 